\title{
Resting-state functional MRI connectivity impact on epilepsy surgery plan and surgical candidacy: prospective clinical work
}

\author{
Varina L. Boerwinkle, MD, ${ }^{1}$ Lucia Mirea, PhD, ${ }^{2}$ William D. Gaillard, MD, ${ }^{3}$ \\ Bethany L. Sussman, PhD, ${ }^{4}$ Diana Larocque, RN, ${ }^{1}$ Alexandra Bonnell, BS, ${ }^{4}$ \\ Jennifer S. Ronecker, MD, ${ }^{5}$ Matthew M. Troester, DO, ${ }^{1}$ John F. Kerrigan, MD, ${ }^{1}$ \\ Stephen T. Foldes, PhD, ${ }^{4}$ Brian Appavu, MD, ${ }^{1}$ Randa Jarrar, MD, ${ }^{1}$ Korwyn Williams, MD, PhD, ${ }^{1}$ \\ Angus A. Wilfong, MD, ${ }^{1}$ and P. David Adelson, MD ${ }^{4}$
}

\begin{abstract}
${ }^{1}$ Division of Pediatric Neurology, ${ }^{2}$ Department of Clinical Research, ${ }^{4}$ Neuroscience Research, and ${ }^{5}$ Division of Pediatric Neurosurgery, Barrow Neurological Institute at Phoenix Children's Hospital, Phoenix, Arizona; and 'Department of Neurology, Children's National Medical Center, Washington, DC
\end{abstract}

OBJECTIVE The authors' goal was to prospectively quantify the impact of resting-state functional MRI (rs-fMRI) on pediatric epilepsy surgery planning.

METHODS Fifty-one consecutive patients ( 3 months to 20 years old) with intractable epilepsy underwent rs-fMRI for presurgical evaluation. The team reviewed the following available diagnostic data: video-electroencephalography $(n=$ 51), structural MRI $(n=51), \operatorname{FDG}-P E T(n=42)$, magnetoencephalography $(n=5)$, and neuropsychological testing $(n=51)$ results to formulate an initial surgery plan blinded to the rs-fMRI findings. Subsequent to this discussion, the connectivity results were revealed and final recommendations were established. Changes between pre- and post-rs-fMRI treatment plans were determined, and changes in surgery recommendation were compared using McNemar's test.

RESULTS Resting-state fMRI was successfully performed in $50(98 \%)$ of 51 cases and changed the seizure onset zone localization in $44(88 \%)$ of 50 patients. The connectivity results prompted 6 additional studies, eliminated the ordering of 11 further diagnostic studies, and changed the intracranial monitoring plan in 10 cases. The connectivity results significantly altered surgery planning with the addition of 13 surgeries, but it did not eliminate planned surgeries $(p=0.003)$. Among the 38 epilepsy surgeries performed, the final surgical approach changed due to rs-fMRI findings in 22 cases $(58 \%)$, including $8(28 \%)$ of 29 in which extraoperative direct electrical stimulation mapping was averted.

CONCLUSIONS This study demonstrates the impact of rs-fMRI connectivity results on the decision-making for pediatric epilepsy surgery by providing new information about the location of eloquent cortex and the seizure onset zone. Additionally, connectivity results may increase the proportion of patients considered eligible for surgery while optimizing the need for further testing.

https://thejns.org/doi/abs/10.3171/2020.1.PEDS19695

KEYWORDS epilepsy surgery; resting-state functional MRI; connectivity; seizure; surgical candidacy; surgical technique

$\mathrm{T}$ HE prevalence of epilepsy is 1 in 26 individuals, ${ }^{9}$ and $30 \%$ of patients are classified as having drugresistant epilepsy (DRE). ${ }^{12,21}$ DRE requires many resources ${ }^{18}$ causes significant disability ${ }^{18}$ and is associated with high mortality.,22 Resective surgery leads to seizure freedom in $40 \%-80 \%$ of patients. ${ }^{24}$ Thus, evaluation is recommended by the American Academy of Neurology and the International League Against Epilepsy. ${ }^{8,10,11,26}$ Despite these recommendations, less than $1 \%$ of DRE patients receive evaluation, and only $25 \%$ of these patients undergo surgery.

The primary determinant of surgical candidacy and

ABBREVIATIONS DRE = drug-resistant epilepsy; EEG = electroencephalography; EPCC = epilepsy patient care conference; ICA = independent component analysis; MEG = magnetoencephalography; RNS = responsive neurostimulation; rs-fMRI = resting-state functional MRI; RSN = resting-state network; SOZ = seizure onset zone; VNS = vagus nerve stimulation.

SUBMITTED November 21, 2019. ACCEPTED January 10, 2020.

INCLUDE WHEN CITING Published online March 20, 2020; DOI: 10.3171/2020.1.PEDS19695. 
postoperative seizure frequency is accurate localization of the seizure onset zone (SOZ), which, when excised or disconnected, should lead to cure. ${ }^{25}$ Recently, several studies have indicated that resting-state functional MRI (rs-fMRI) localizes where seizures originate in the brain, ${ }^{2-4}$ and its implementation improves surgical outcome. ${ }^{5}$ Agreement of rs-fMRI SOZ and intracranial electroencephalography (EEG) SOZ localization is $90 \% .{ }^{4}$ Quantification of resection with respect to preoperative rs-fMRI SOZ localization has shown that when at least $80 \%$ of the rs-fMRI SOZ is removed, Engel class I seizure outcomes at the 2-year follow-up occur in $97 \%$, compared with only $5 \%$ in those without equivalent removal, demonstrating a $94 \%$ accuracy for the true SOZ. ${ }^{2-4}$

In contradistinction to these prior studies designed to verify the validity of rs-fMRI SOZ, there are no studies, to our knowledge, quantifying the impact of rs-fMRI on increasing surgical candidacy.

This study's overarching aim was to prospectively quantify the impact of rs-fMRI on the epilepsy patient care conference (EPCC) impression of surgical candidacy and surgical plan in children with DRE. We aimed to compare rates of SOZ localization by rs-fMRI with other diagnostics, as interpreted by the EPCC. We focused on comparing the EPCC impression of the rs-fMRI network mapping for motor, language, and other cortical networks with other diagnostic information, and whether rs-fMRI added new information that changed the consensus of the SOZ location, planning for additional studies, or surgical plan. Finally, we examined how rs-fMRI specifically impacted EPCC planning for neuromodulation by vagus nerve stimulation (VNS) and responsive neurostimulation (RNS).

\section{Methods}

The local institutional review board granted approval for the retrospective review of the clinical impact of rsfMRI utilized prospectively to guide neurosurgery. Resting-state fMRI became the standard evaluation for epilepsy surgery in May 2017 at the study institution, given the evidence that surgical targeting of the rs-fMRI-detected SOZ improved surgical outcomes with a good safety profile in the pediatric general epilepsy population. ${ }^{4}$ Therefore, no additional consent was deemed necessary for rsfMRI.

Fifty-one consecutive patients with DRE underwent rsfMRI as part of the presurgical evaluation, followed by the EPCC meeting. Table 1 describes the cohort. The mean patient age was 11.4 years (SD 5.3 years). Patients who were determined to require conscious sedation received a propofol infusion, as is the institution's standard of care for pediatric patients who cannot cooperate with an awake MRI. Resting-state fMRI was obtained regardless of the timing of the last known seizure event.

\section{Inclusion Criteria}

The primary determinate of inclusion was based on the treating epileptologist's decision that the patient had DRE and should receive epilepsy surgery evaluation. Rapid generalization of epileptiform activity from a focal epi-
TABLE 1. Characteristics of study patients and surgeries

\begin{tabular}{lc}
\hline \multicolumn{1}{c}{ Characteristic } & Value \\
\hline Age, yrs & \\
Mean (SD) & $11.4(5.3)$ \\
Median (IQR) & $11.3(8.4-11.5)$ \\
\hline Sex & \\
Male & $23(45)$ \\
Female & $28(55)$ \\
\hline Handedness & \\
Rt & $40(78)$ \\
Lt & $6(12)$ \\
Nonhanded & $5(10)$ \\
\hline ILAE epilepsy cause & \\
Brain structure abnormalities & $25(49)$ \\
Genetics/tuberous sclerosis complex & $4(8)$ \\
Other (metabolic, infectious, immune, or unknown) & $22(43)$ \\
\hline Lobe location & \\
Negative MRI & $13(25)$ \\
Frontal & $6(12)$ \\
Temporal & $11(22)$ \\
Parietal & $6(12)$ \\
Extends to >1 lobe unilaterally & $8(16)$ \\
Bilateral, extensive & $5(10)$ \\
Other & $2(4)$ \\
\hline Surgery received & \\
No surgery & $12(24)$ \\
VNS & $4(8)$ \\
RNS & $7(14)$ \\
Laser & $11(22)$ \\
Craniectomy & $17(33)$ \\
\hline &
\end{tabular}

ILAE = International League Against Epilepsy.

Values represent the number of patients (\%) unless stated otherwise.

leptogenic focus may appear generalized on surface EEG and/or by semiology; thus, generalized epilepsy was not considered as an exclusion parameter.

\section{MRI Sequence}

Images were acquired on a 3T MRI unit (Ingenuity, Philips Medical Systems) equipped with a 32-channel head coil. Resting-state fMRI parameters included TR $2000 \mathrm{msec}$, TE $30 \mathrm{msec}$, matrix size $80 \times 80$, flip angle $80^{\circ}$, number of slices 46 , slice thickness $3.4 \mathrm{~mm}$ with no gap, in-plane resolution $3 \times 3 \mathrm{~mm}$, interleaved acquisition, and number of total volumes 600 , in two 10-minute runs, totaling 20 minutes. For anatomical reference, a T1weighted turbo field echo whole-brain MRI sequence was obtained using the following parameters: TR $9 \mathrm{msec}$, TE $4 \mathrm{msec}$, flip angle $8^{\circ}$, slice thickness $0.9 \mathrm{~mm}$, and in-plane resolution $0.9 \times 0.9 \mathrm{~mm}$.

\section{MRI Analysis}

Importantly, the rs-fMRI SOZ determination in this same cohort of patients was determined as part of a prior study. ${ }^{2}$ That study utilized a system of three blinded reviewers to inspect the rs-fMRI images, confirming the rsfMRI SOZ. In contrast, the current study is not focused on 
these previously determined imaging and seizure-based outcome quantifications; the reader is directed to the prior study for the full details of the image analysis methodology and surgical outcomes.

Briefly, analysis of rs-fMRI data was carried out using the Oxford Centre FMRIB (Functional MRI of the Brain) Software Library tool MELODIC, ${ }^{1}$ as previously reported..$^{2,4,20}$ Standard preprocessing steps were applied: the first 5 volumes were deleted to remove T1 saturation effects, high-pass filtering at 100 seconds, slice time correction, spatial smoothing of 1-mm full-width at halfmaximum, and motion corrected by MCFLIRT, ${ }^{15}$ with nonbrain structures removed.

Individual functional scans were registered to the patient's high-resolution anatomical scan using linear registration ${ }^{16}$ and optimized using boundary-based registration. ${ }^{13}$ Individual rs-fMRI data sets then underwent independent component analysis (ICA) as previously reported. ${ }^{4}$ Resulting components were categorized as noise, healthy resting-state network (RSN), atypical RSN, or rs-fMRI SOZ by prior detailed criteria. ${ }^{2,4,14}$ The rs-fMRI results presented to the team were images of the expected RSNs (motor, language, and visual networks and rs-fMRI SOZ).

\section{Resting-State SOZ Localization}

Resting-state fMRI SOZs are differentiated from typical RSNs and noise based on previously published spatial and temporal features.

Resting-state fMRI SOZ spatial features are as follows.

1) Must be primarily located within the gray matter, but not in the RSN spatial pattern.

2) May have a bull's-eye pattern: two or more spatially overlapping abnormal neuronal independent components and may have surrounding and/or overlapping atypical RSN(s). If a bull's-eye pattern is present, then the area of overlap between the abnormal neuronal independent components is considered the most abnormal area and is the rs-fMRI SOZ.

3) May have an alternating activation-deactivation pattern that is not in typical noise zones (such as arteries, veins, or outside the brain tissue) or detected only in a single slice, or alternating slices or streaks in the phaseencoding direction (noise related to the MRI sequence, echo-planar imaging susceptibility, or multiband acceleration).

4) May have extension toward ventricles through the white matter.

5) May have highly irregular borders. ${ }^{2}$

Resting-state fMRI SOZ temporal features are as follows.

1) Must contain frequency $>0.073 \mathrm{~Hz}$.

2) If a downstream RSN is overlapping or spatially contiguous, then the rs-fMRI SOZ must contain a frequency greater than the RSN.

3) May have irregular oscillation, such as sharply contoured bursts of irregular runs of faster frequency than baseline with or without gradual return to normal (period change greater than half baseline period between cycles). ${ }^{2}$
TABLE 2. Seizure onset zone localizing tests

\begin{tabular}{lcc}
\hline \multicolumn{1}{c}{ Test } & No. of Pts (\%) & Localization Rate $(95 \% \mathrm{Cl})^{*}$ \\
\hline Seizure semiology & $24 / 51(47)$ & $0.47(0.33-0.62)$ \\
\hline MRI positive & $40 / 51(78)$ & $0.78(0.65-0.89)$ \\
\hline EEG & $42 / 51(82)$ & $0.82(0.69-0.92)$ \\
\hline PET localizing & $26 / 42(62)$ & $0.62(0.46-0.76)$ \\
\hline MEG localizing & $3 / 5(60)$ & $0.60(0.15-0.95)$ \\
\hline Resting-state fMRI & & \\
$\quad$ Generalized & $4 / 51(8)$ & $0.92(0.81-0.98)$ \\
$\quad$ Localizing & $46 / 51(90)$ & \\
Poor-quality data & $1 / 51(2)$ & \\
\hline
\end{tabular}

$\mathrm{Pt}=$ patient.

* Confidence intervals were computed using the exact Clopper-Pearson method.

\section{Epilepsy Patient Care Conference}

The EPCC team consisted of pediatric epileptologists, neurosurgeons, neuropsychologists, and neuroradiologists. During the EPCC, patient information that was initially presented included handedness, epilepsy characteristics, etiology, current and past treatments, video-EEG tracings with seizure semiology, and other seizure test results (Table 2), and non-rs-fMRI brain mapping test results. Resting-state fMRI results were presented only after a surgical consensus was agreed on by the EPCC.

\section{Blinding}

The rs-fMRI rater (V.L.B.) was initially blinded to patient history, etiology, treatments, semiology, and seizurelocalizing test results at the time of the rs-fMRI interpretation. Anatomical MRI, without radiological interpretation, was used for registration in rs-fMRI analysis; thus, gross differences from normal were potentially visible. As this was a clinically ordered study, the rs-fMRI rater reviewed patient information after blinded interpretation to ensure that the results made clinical sense for the patient. The blinded rs-fMRI interpretations did not change after the final nonblinded review.

The EPCC members were blinded to rs-fMRI results prior to the conference (except for the treating epileptologists, who had previously received the rs-fMRI results but did not reveal them to the team members who formulated the pre-rs-fMRI plan). The rs-fMRI rater was present, though not contributing. The rater read the rs-fMRI clinical report verbatim and answered clarification questions from the team without participating in the EPCC's surgical plan determination. The verbalized treatment plans were prospectively recorded by the epilepsy care coordinator nurse before and after presentation of rs-fMRI results.

\section{Localizing Tests}

The number of patients who received each type of seizure-localizing modality are delineated in Table 2 . These included seizure semiology, high-resolution 3T structural MRI, ictal video-EEG, PET, and magnetoencephalography (MEG). In Table 2, the second column denotes how 
often the modality yielded localizing results. Tests were determined to be "localizing" if the inclusion of their data changed the EPCC's hypothesized SOZ (see SOZ Localization below for further details). MEG was infrequently performed due to a lack of local availability. SPECT was not performed due to local nuclear medicine and radiation safety regulations that limited practice. Treatment plan elements that were discussed were recorded and are delineated in Table 1. For each modality, the localization rate and corresponding $95 \%$ confidence interval were computed to enable comparisons of localization between modalities. Agreement between rs-fMRI and all other modalities was assessed using McNemar's test (Table 2). ${ }^{19}$

\section{Treatment Change Analysis}

The consensus of two blinded analysis team members determined whether inclusion of rs-fMRI results changed the treatment strategy. The plan was for a third member to make a determination if consensus was not reached by the first two, which did not occur. The analysis team reviewed EPCC-recorded notes 3-12 months after EPCC presentation. The analysis team was blinded to patient identity and reviewed patients in the order of presentation date. The analysis team consisted of the EPCC nurse coordinator (D.L.), a neurosurgeon (J.S.R.), and a neurologist (V.L.B.), who did not participate in EPCC surgical planning. The instructions provided to the analysis team were to determine whether rs-fMRI results changed the EPCC impression of the following categorical variables.

\section{SOZ Location}

The study analysis team noted the following.

- If the rs-fMRI report indicated an SOZ was detected by rs-fMRI.

- If rs-fMRI results changed, 1) whether or not the patient should undergo a phase II or III evaluation, 2) the regions planned for intracranial electrodes, 3) the decision to perform cortical mapping, and 4) the surgical modality (laser thermocoagulation vs open craniotomy). Determinations of change were not based on quantification of SOZ size or specific location (e.g., lobe) differences. Rather, the analysis team was asked to determine if the EPCC considered the rs-fMRI-detected SOZ clinically meaningful, and whether the location of the SOZ hypothesized on pre-rs-fMRI was altered by the rs-fMRI findings or stayed the same.

\section{Eloquent Mapping}

The study analysis team determined the following.

- If rs-fMRI identified the language, vision, and/or motor networks.

- If the rs-fMRI network(s) location altered surgical plans to avoid morbidity related to the network function.

- If invasive brain mapping was cancelled post-rs-fMRI or modified the areas to be studied by intracranial EEG.

\section{Neuromodulation}

The analysis team noted the following.
- If the pre-rs-fMRI EPCC plan offered VNS or RNS, and if this plan was being considered due to either the patient having generalized or poorly localized epilepsy, or the SOZ location was not amenable to surgery.

- If the rs-fMRI SOZ pattern was reported as generalized or poorly localized, meaning that the majority of the brain has equally abnormal connectivity and is declared supportive of generalized epilepsy or focal epilepsy.

- If the post-rs-fMRI surgical plan changed from VNS (extracranial) to RNS (intracranial), or from VSN or RNS to a disconnective/destructive surgical approach.

- If the post-rs-fMRI EPCC impression of the rs-fMRI $\mathrm{SOZ}$ is congruent with the plan to offer VNS, meaning both are indicating generalized or poorly localized epilepsy.

- If the post-rs-fMRI EPCC impression of the planned responsive neurostimulator location was supported by the focal rs-fMRI SOZ location.

\section{Surgical Candidacy}

The analysis team noted the following.

- If the pre-rs-fMRI and post-rs-fMRI plan changed, and how so.

- If surgery was recommended.

- If surgery was recommended, then what type of surgery was recommended (i.e., open craniotomy-based, minimally invasive laser ablation, RNS, VNS, or other).

\section{Statistical Analysis}

Baseline demographics and clinical factors were summarized using counts and percentages for categorical variables and the mean and standard deviation or median and interquartile range for quantitative measures. The proportion of cases with rs-fMRI localization was calculated with corresponding 95\% confidence intervals. McNemar's test compared localization rates between rs-fMRI and other modalities.

The proportion of patients with changes in localization and treatment plans due to rs-fMRI were calculated. Agreement of surgery recommendations before and after rs-fMRI evaluation was quantified by the kappa coefficient and $95 \%$ confidence intervals and compared using McNemar's test. Statistical analyses were performed using the software package SAS (version 9.4, SAS Institute). All statistical tests were 2-sided, with significance evaluated at the $5 \%$ level.

\section{Results}

Of the 51 consecutive patients who underwent rs-fMRI, 39 required conscious sedation. No safety-related events or patient morbidity occurred due to rs-fMRI. Resting-state fMRI did not detect the expected networks in 1 patient, who required more than the typical dosage of sedation to reduce patient motion. This patient did eventually have surgery (as accounted for in Table 1) but was excluded from further rs-fMRI analysis. The remaining 50 patients had less than $1 \mathrm{~mm}$ of head motion in any direction during scanning and had detection of the major cortical networks. Demographics, the presence and location of MRI-visible lesions, and type of surgery performed are presented in 
TABLE 3. Changes in treatment plan due to rs-fMRI findings

\begin{tabular}{lr}
\hline \multicolumn{1}{c}{ Change in Treatment Plan } & No. of Pts (\%) \\
\hline Brain function \& seizure mapping & $44 / 50(88)$ \\
Altered SOZ location & $46 / 50(92)$ \\
rs-fMRI only source of objective language lateralization & $9 / 50(18)$ \\
Change in location of language function & $12 / 50(24)$ \\
Change in motor or visual cortical function location & $5 / 25(20)$ \\
Diagnostic/therapeutic strategy & \\
In 25 pts w/o other studies planned pre-rs-fMRI & $3 / 25(12)$ \\
Additional studies performed & $11 / 25(44)$ \\
In 25 pts w/ further studies planned pre-rs-fMRI & $10 / 25(40)$ \\
No change & $1 / 25(4)$ \\
Cancelled study & $47 / 50(94)$ \\
Altered intracranial monitoring location/strategy & \\
Added new study & $10 / 11(91)$ \\
Altered patient \& family counseling & $13 / 50(26)$ \\
Surgical decision & $6 / 13(46)$ \\
Helped decide to get modulation (VNS, RNS) & $7 / 13(54)$ \\
Family consent to surgery & $37 / 50(74)$ \\
Team decision post-rs-fMRI: no surgery & $6 / 37(16)$ \\
Family declined & $31 / 37(84)$ \\
Family consented & $22 / 38(58)$ \\
Team decision post-rs-fMRI: surgery & $8 / 29(18)$ \\
Family declined & \\
Family consented &
\end{tabular}

Table 1. Patient-level data are presented in Supplementary Table A.

\section{Seizure-Localizing Test Comparison}

Resting-state fMRI provided impactful SOZ localizing information for a significantly greater proportion of patients than did seizure semiology or PET (McNemar's test, $\mathrm{p}<0.002$; Table 2). Localization rates of other seizurelocalizing tests (anatomical MRI, EEG, and MEG) were also lower than rs-fMRI, but the 95\% CIs overlapped, and no statistical significance was detected. Note that this had no bearing on the uniqueness of the SOZ by any modality, only that the EPCC regarded the test as helpful in localization.

\section{Treatment Plan Changes}

The 50 patients who had successful rs-fMRI acquisition with treatment plan changes are delineated in Tables 3 and 4.

\section{Seizure Onset Zone}

The EPCC-hypothesized location of the SOZ was altered pre- to post-rs-fMRI in $44(88 \%)$ of 50 patients, which subsequently impacted various aspects of surgical planning. Among the 25 patients with additional studies planned, rs-fMRI altered the location of intracranial electrode placement in 10 (40\%). Among the 38 resections or disconnection surgeries, rs-fMRI changed the EPCC's area intended for surgical removal or responsive neuro- stimulator placement by rs-fMRI SOZ in $22(58 \%)$ of 38 patients.

\section{Eloquent Network Mapping}

Resting-state fMRI was the only source of objective language lateralization information in 46 (92\%) of 50 patients and changed the hypothesized location of language function (based on the handedness-hemispheric language dominance pattern ${ }^{17}$ ) in 9 patients $(18 \%)$. The other $8 \%$ had task-based fMRI language testing. Resting-state fMRI also changed the prior hypothesized location of the motor and visual cortical function, based primarily on the EPCC's neuroanatomical assumptions, in 12 patients $(24 \%)$.

\section{Altering Additional Testing}

Overall, rs-fMRI resulted in a net reduction of additional studies in $5(10 \%)$ of 50 patients. In patients with further studies planned prior to revealing the rs-fMRI findings, 11 (44\%) of 25 studies were cancelled, and a new study was added in 1 (4\%) of 25 patients. Among the cancelled studies, 7 were intracranial EEG for SOZ localization (overall 18\% reduction in intracranial EEG studies), 2 were MEG, and 2 were intracranial brain stimulation mapping. One MEG study was added after the rs-fMRI findings were revealed in the group with planned further studies. Among 25 patients without other studies planned prior to revealing the rs-fMRI results, additional studies were performed in 5 (20\%) of 25 patients. 
TABLE 4. Resting-state fMRI impact on the team's decision to offer surgery for the patient

\begin{tabular}{|c|c|c|c|c|c|}
\hline \multirow[b]{2}{*}{ Pre-rs-fMRI } & \multicolumn{2}{|c|}{ Post-rs-fMRI } & \multicolumn{2}{|c|}{$p$ Value } & \multirow{2}{*}{$\begin{array}{c}\text { Kappa } \\
\text { Coefficient } \\
(95 \% \mathrm{Cl})\end{array}$} \\
\hline & $\begin{array}{l}\text { Offered } \\
\text { Surgery }\end{array}$ & $\begin{array}{l}\text { No Surgery } \\
\text { Offered }^{*}\end{array}$ & $\begin{array}{l}\text { McNemar's } \\
\text { Test }\end{array}$ & $\begin{array}{c}\text { Fisher Exact } \\
\text { Test }\end{array}$ & \\
\hline Offered surgery $(n=24)$ & 24 & 0 & \multirow{2}{*}{0.003} & \multirow{2}{*}{$<0.0001$} & \multirow{2}{*}{$0.49(0.28-0.69)$} \\
\hline No surgery offered $(n=26)$ & 13 & 13 & & & \\
\hline
\end{tabular}

\section{Surgical Decisions}

The flow of EPCC decisions is shown in Fig. 1, which differentiates between surgical procedures recommended and those completed. Of the 50 patients with successful rs-fMRI, the decision to offer surgery was changed after rs-fMRI for 13 (26\%) of 50 patients who were initially not considered for surgery pre-rs-fMRI (Table 4). Of these 13,8 went through with the surgery. Thus, $31 \%$ of patients (8/26) had surgery who otherwise would not have undergone surgery. All 24 patients identified as surgical candidates pre-rs-fMRI remained surgical candidates post-rsfMRI.

\section{Neuromodulation}

Overall, rs-fMRI influenced in some way the treatment of 10 of 11 patients considered for neuromodulation by VNS or RNS. Four patients were considered for and re- ceived VNS. Of these 4 patients, rs-fMRI supported the recommendation, as the rs-fMRI SOZ pattern was generalized, indicating that a focal treatment may not be effective. Epilepsy surgery planning is currently based on multimodality data. The level of utility of confirmatory data may not be as great as divergent data that lead to an alternative plan. Nonetheless, if all tested modalities confirm VNS to be the best option for the patient, this would be considered a more assured decision than if the modalities provided divergent results. Additionally, in 2 of these patients who underwent VNS, MEG was considered prers-fMRI but was cancelled post-rs-fMRI due to a lack of focal SOZ by rs-fMRI.

In total, 7 patients received RNS. Three of these decisions were made post-rs-fMRI, as rs-fMRI was a major determinant in the EPCC plan for responsive neurostimulator lead placement location. Of the remaining 4 patients,

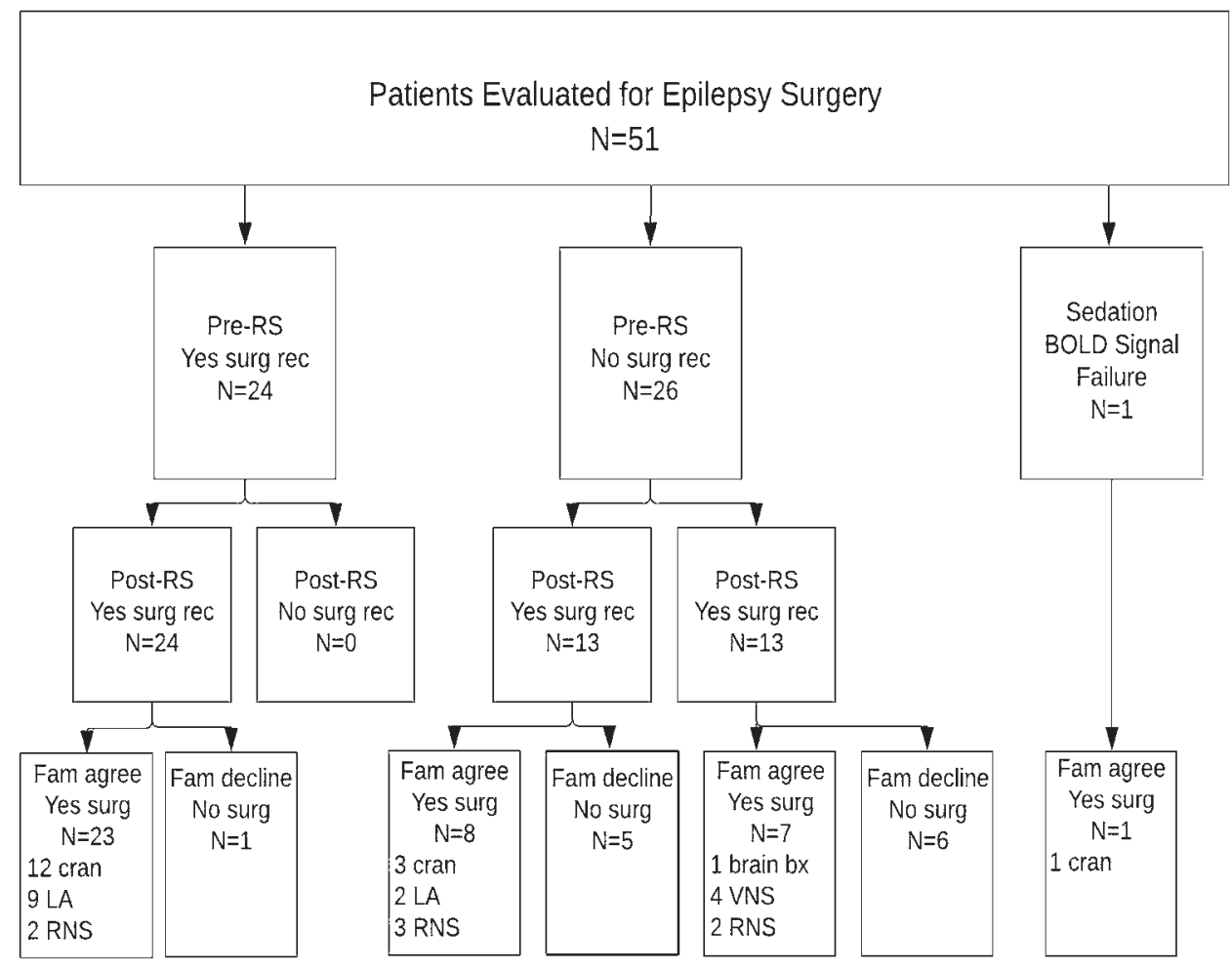

FIG. 1. Patient flowchart. Diagrams the pre- to post-rs-fMRI EPCC team recommendations and if the family agreed or declined the plan offered. Surgical approach completed is quantified. bx = biopsy; cran = craniectomy; fam = family; LA = laser ablation; rec = recommended; RS = rs-fMRl; surg = surgery. 
the rs-fMRI SOZ altered the planned lead placement in 1 , supported prior planned lead placement in 3 , and was nonlocalizing in 1.

Epilepsy surgery teams often evaluate children for resective surgery and then may determine that resection is not safe or perhaps the seizure focus hypothesis is too weak for a destructive surgical approach, opting then for neuromodulation. The comprehensive inclusion of both groups-resective and neuromodulation-improved understanding of whether rs-fMRI is helpful in such situations and was clinically informative to the local team.

\section{Discussion}

\section{SOZ Localization and Surgical Approach}

We prospectively quantified the impact of rs-fMRI on surgical planning in children with DRE. The addition of rs-fMRI resulted in $13(50 \%)$ of 26 patients becoming surgical candidates who would not have been so without rs-fMRI. We suggest that rs-fMRI influenced EPCC decisions primarily through alteration of $\mathrm{SOZ}$ localization ( $88 \%$ of 50 ). In turn, this led to changes in planned spatial coverage for intracranial EEG (40\% of 25) and/or extent of resection or location of responsive neurostimulator lead placement in the other patients. Resting-state fMRI triggered further localization studies in patients who were not considered surgical candidates pre-rs-fMRI (5 [20\%] of $25)$, thus increasing surgical candidacy.

\section{Eloquent Network Mapping}

Resting-state fMRI also impacted the EPCC's localization of eloquent networks important to avoid in epilepsy surgical planning. Resting-state fMRI was the only modality of language mapping presented at the conference for most patients (92\%) and changed the hypothesized language network location in $18 \%$ of the 50 patients. The decision to accept rs-fMRI language mapping could be due to a combination of an awareness of evidence supporting rs-fMRI accuracy in language lateralization in $\mathrm{DRE}^{6}$ and a patient's inability to participate in task-based tests influencing clinical utilization of rs-fMRI.

\section{Additional Studies}

The net reduction of additional extraoperative direct electrical stimulation mapping sessions provides a potential cost-saving measure (often phase II is already in place for other reasons, and cortical mapping itself takes a few hours of physician time), and the potential means to substitute a noninvasive, comparatively low-risk test for surgical planning.

Conversely, rs-fMRI influences decisions to perform additional studies. Importantly, the majority of patients for whom a study was added had not been considered candidates for surgical intervention before rs-fMRI review. After review of the rs-fMRI findings, the EPCC concluded that these patients may be surgical candidates, and a confirmatory test (usually MEG or stereo-EEG) was ordered to corroborate the findings. Taken together, these results suggest that rs-fMRI review can also facilitate selective use of mapping studies to positively influence surgical candidacy and planning.

\section{Neuromodulation}

We did not find that rs-fMRI added novel information to the VNS evaluations, except to reduce the number of MEG scans ordered. Resting-state fMRI was helpful overall in increasing candidacy for RNS by SOZ detection.

\section{Future Directions}

National guidelines do not yet incorporate rs-fMRI into recommendations. ${ }^{23}$ Studies such as the current work's quantification of rs-fMRI's impact on surgical candidacy and surgical decisions may influence such guidelines, thereby influencing other centers to incorporate rs-fMRI into clinical practice.

While this single-institution study was positive, it is limited by the size of the patient cohort and is from a single institution. Multi-institutional rs-fMRI studies are needed to understand the impact of rs-fMRI on integration into clinical care. Notably, similar to all other seizure localization modalities, this approach also still relies on pattern recognition of rs-fMRI-detected SOZ. ${ }^{2-4}$ Future studies may also compare prior institutional surgical outcomes to those after incorporating rs-fMRI. Differences in local center availability of MEG and SPECT could alter nuances in the incorporation pattern of rs-fMRI.

\section{Conclusions}

Epilepsy surgical candidacy increased by $26 \%$ after rs-fMRI results changed surgical team decisions. The connectivity results impacted the surgical team's plan regarding which areas were selected to be monitored by intracranial EEG and altered the final surgical approach. Resting-state fMRI results led to a net reduction in additional studies ordered for epilepsy surgery evaluation. Connectivity results may lead to increased opportunities for potential cure or amelioration of epilepsy.

\section{References}

1. Beckmann CF, Smith SM. Probabilistic independent component analysis for functional magnetic resonance imaging. IEEE Trans Med Imaging. 2004;23(2):137-152.

2. Boerwinkle VL, Cediel EG, Mirea L, et al. Network-targeted approach and postoperative resting-state functional magnetic resonance imaging are associated with seizure outcome. Ann Neurol. 2019;86(3):344-356.

3. Boerwinkle VL, Foldes ST, Torrisi SJ, et al. Subcentimeter epilepsy surgery targets by resting state functional magnetic resonance imaging can improve outcomes in hypothalamic hamartoma. Epilepsia. 2018;59(12):2284-2295.

4. Boerwinkle VL, Mohanty D, Foldes ST, et al. Correlating resting-state functional magnetic resonance imaging connectivity by independent component analysis-based epileptogenic zones with intracranial electroencephalogram localized seizure onset zones and surgical outcomes in prospective pediatric intractable epilepsy study. Brain Connect. 2017;7(7):424-442.

5. Carboni M, Rubega M, Iannotti GR, et al. The network integration of epileptic activity in relation to surgical outcome. Clin Neurophysiol. 2019;130(12):2193-2202.

6. Desai VR, Vedantam A, Lam SK, et al. Language lateralization with resting-state and task-based functional MRI in pediatric epilepsy. J Neurosurg Pediatr. 2018;23(2):171-177.

7. Engel J Jr. What can we do for people with drug-resistant 
epilepsy? The 2016 Wartenberg Lecture. Neurology. 2016;87(23):2483-2489.

8. Engel J Jr, McDermott MP, Wiebe S, et al. Early surgical therapy for drug-resistant temporal lobe epilepsy: a randomized trial. JAMA. 2012;307(9):922-930.

9. England MJ, Liverman CT, Schultz AM, Strawbridge LM. Summary: a reprint from epilepsy across the spectrum: promoting health and understanding. Epilepsy Curr. 2012;12(6):245-253.

10. Fiest KM, Sajobi TT, Wiebe S. Epilepsy surgery and meaningful improvements in quality of life: results from a randomized controlled trial. Epilepsia. 2014;55(6):886-892.

11. Fountain NB, Van Ness PC, Bennett A, et al. Quality improvement in neurology: Epilepsy Update Quality Measurement Set. Neurology. 2015;84(14):1483-1487.

12. French JA. Refractory epilepsy: clinical overview. Epilepsia. 2007;48(suppl 1):3-7.

13. Greve DN, Fischl B. Accurate and robust brain image alignment using boundary-based registration. Neuroimage. 2009;48(1):63-72.

14. Griffanti L, Salimi-Khorshidi G, Beckmann CF, et al. ICAbased artefact removal and accelerated fMRI acquisition for improved resting state network imaging. Neuroimage. 2014;95:232-247.

15. Jenkinson M, Bannister $\mathrm{P}$, Brady $M$, Smith $\mathrm{S}$. Improved optimization for the robust and accurate linear registration and motion correction of brain images. Neuroimage. 2002;17(2):825-841.

16. Jenkinson M, Smith S. A global optimisation method for robust affine registration of brain images. Med Image Anal. 2001;5(2):143-156.

17. Knecht S, Deppe M, Dräger B, et al. Language lateralization in healthy right-handers. Brain. 2000;123(Pt 1):74-81.

18. Laxer KD, Trinka E, Hirsch LJ, et al. The consequences of refractory epilepsy and its treatment. Epilepsy Behav. 2014;37:59-70.

19. McNemar Q. Note on the sampling error of the difference between correlated proportions or percentages. Psychometrika. 1947;12(2):153-157.

20. Mongerson CRL, Jennings RW, Borsook D, et al. Restingstate functional connectivity in the infant brain: methods, pitfalls, and potentiality. Front Pediatr. 2017;5:159.

21. Picot MC, Baldy-Moulinier M, Daurès JP, et al. The prevalence of epilepsy and pharmacoresistant epilepsy in adults: a population-based study in a Western European country. Epilepsia. 2008;49(7):1230-1238.

22. Sillanpää M, Shinnar S. Long-term mortality in childhoodonset epilepsy. N Engl J Med. 2010;363(26):2522-2529.

23. Szaflarski JP, Gloss D, Binder JR, et al. Practice guideline summary: use of fMRI in the presurgical evaluation of patients with epilepsy: report of the Guideline Development, Dissemination, and Implementation Subcommittee of the American Academy of Neurology. Neurology. 2017;88(4):395-402.
24. Tonini C, Beghi E, Berg AT, et al. Predictors of epilepsy surgery outcome: a meta-analysis. Epilepsy Res. 2004;62(1):7587.

25. West S, Nevitt SJ, Cotton J, et al. Surgery for epilepsy. Cochrane Database Syst Rev. 2019;6:CD010541.

26. Wiebe S, Blume WT, Girvin JP, Eliasziw M. A randomized, controlled trial of surgery for temporal-lobe epilepsy. $N$ Engl J Med. 2001;345(5):311-318.

\section{Disclosures}

The authors report no conflict of interest concerning the materials or methods used in this study or the findings specified in this paper.

\section{Author Contributions}

Conception and design: Boerwinkle, Gaillard, Larocque, Ronecker, Troester, Kerrigan, Foldes, Appavu, Jarrar, Williams, Wilfong, Adelson. Acquisition of data: Boerwinkle, Mirea, Larocque, Ronecker, Troester, Kerrigan, Appavu, Jarrar, Williams, Wilfong, Adelson. Analysis and interpretation of data: Boerwinkle, Mirea, Sussman, Larocque, Bonnell, Ronecker, Troester, Wilfong, Adelson. Drafting the article: Boerwinkle, Gaillard, Sussman, Bonnell, Troester, Kerrigan, Foldes, Williams, Wilfong, Adelson. Critically revising the article: Boerwinkle, Mirea, Gaillard, Sussman, Bonnell, Ronecker, Troester, Foldes, Appavu, Jarrar, Williams, Wilfong, Adelson. Reviewed submitted version of manuscript: all authors. Approved the final version of the manuscript on behalf of all authors: Boerwinkle. Statistical analysis: Boerwinkle, Mirea, Sussman, Appavu. Study supervision: Boerwinkle.

\section{Supplemental Information}

\section{Online-Only Content}

Supplemental material is available with the online version of the article.

Supplementary Table A. https://thejns.org/doi/suppl/10.3171/ 2020.1.PEDS19695.

\section{Correspondence}

Varina L. Boerwinkle: Barrow Neurological Institute at Phoenix Children's Hospital, Phoenix, AZ. vboerwinkle@ phoenixchildrens.com. 